\title{
Long non-coding RNA NEAT1 contributes to lipopolysaccharide-induced inflammation and apoptosis of human middle ear epithelial cells via regulating the miR-301b-3p/TLR4 axis
}

\author{
ZHUOHUI LIU*, TAO LU* ${ }^{*}$, SHUMIN LIU* , FAN ZHANG, JINXIONG YANG, \\ SHUMIN DAI, BIAO RUAN and RUIQING LONG \\ Department of Otolaryngology, The First Affiliated Hospital of Kunming \\ Medical University, Kunming, Yunnan 650032, P.R. China
}

Received August 23, 2020; Accepted March 22, 2021

DOI: $10.3892 /$ etm.2021.10795

\begin{abstract}
Acute otitis media (AOM) is a common infectious disease in children that is accompanied by signs and symptoms of middle ear inflammation and infection. Previous studies have shown that the long non-coding (lnc)RNA nuclear-enriched abundant transcript 1(NEAT1) participates in various inflammatory conditions and plays an important regulatory role. The focus of the present study was the biological function of NEAT1 and underlying molecular mechanism in lipopolysaccharide (LPS)-induced human middle ear epithelial cells (HMEECs). The expression of NEAT1, miR-301b-3p and toll-like receptor 4 (TLR4) protein were determined by reverse transcription-quantitative PCR and western blot assays, respectively. Dual-luciferase reporter assay was performed to investigate the combination of miR-301b-3p and NEAT1 or TLR4. In addition, cell viability, apoptosis and the levels of pro-inflammatory factors (IL-1 $\beta$, TNF- $\alpha$ and IL-6) were measured by Cell Counting Kit- 8 assay, flow cytometry and ELISA, respectively. Cell viability was significantly decreased, whereas apoptosis and inflammation were increased in LPS-stimulated HMEECs. Functional analyses demonstrated that NEAT1 was upregulated following LPS treatment, whereas knockdown of NEAT1 significantly increased cell viability and alleviated apoptosis and inflammation. Mechanistically, NEAT1
\end{abstract}

Correspondence to: Dr Ruiqing Long, Department of Otolaryngology, The First Affiliated Hospital of Kunming Medical University, 295 Xichang Road, Kunming, Yunnan 650032, P.R. China E-mail: longrqent@163.com

*Contributed equally

Key words: acute otitis media, nuclear-enriched abundant transcript 1, miR-301b-3p, toll-like receptor 4, human middle ear epithelial cells directly bound to and negatively regulated miR-301b-3p expression, whereas miR-301b-3p inhibitors abolished the inhibitory effect of NEAT1 knockdown on cell apoptosis and inflammation. As a target of miR-301b-3p, TLR4 was regulated by NEAT1 and miR-301b-3p. TLR4 overexpression alleviated NEAT1 silencing-induced inflammatory suppression. Rescue experiments demonstrated that NEAT1 promoted TLR4 expression by inhibiting miR-301b-3p. Collectively, the results of the present study suggested that NEAT1 may attenuate LPS-induced inflammation and apoptosis in HMEECs by modulating the miR-301b-3p/TLR4 axis, and may provide a new therapeutic target for the clinical treatment of AOM.

\section{Introduction}

Acute otitis media (AOM) is one of the most common infectious diseases in childhood caused by bacteria (1), such as Streptococcus pneumoniae, non-typeable Haemophilus influenzae (H. influenzae) and Moraxella catarrhalis (2). AOM is characterized by the presence of middle ear effusion together with an acute onset of signs and symptoms caused by middle ear inflammation. The pathophysiology, pathogenesis and progression of AOM are associated with multiple factors, such as pathogenic bacteria, reactive oxygen species and inflammatory cytokines (3). Currently, due to the extensive use of antibiotics, which may cause resistance of AOM to treatment, $10-20 \%$ of children may experience continuous recurrences of otitis media and long-term deafness (4). Therefore, identifying effective therapies is crucial for the prevention and treatment of AOM.

Long non-coding (lnc)RNAs are a group of heterogeneous non-coding RNAs with a length of $>200$ nucleotides, which are widely distributed in the genome (5). Previous research has shown that IncRNAs play an important role in transcription regulation, maintenance of genomic integrity, alternative splicing, gene chromatin rearrangement $(6,7)$ and various pathophysiological processes $(8,9)$. Several studies have revealed that lncRNAs are implicated in immune defense against bacterial 
infections, involving Salmonella, Mycobacterium bovis and Mycobacterium tuberculosis (10-12). The lncRNA nuclear-enriched abundant transcript 1 (NEAT1) has two isoforms (short isoform NEAT1-1 and long isoform NEAT1-2), which are associated with the prognosis of human tumors (13). In addition, NEAT1 has been reported to be involved in a number of inflammatory diseases. It has been reported that overexpression of NEAT1 protected cardiomyocytes from $\mathrm{As}_{2} \mathrm{O}_{3}$-induced injury (14), whereas other studies re-ported that downregulating NEAT1 inhibited inflammation, fibrosis and apoptosis (15-17). In addition, NEAT1 was found to be upregulated by lipopolysaccharide (LPS) $(15,18)$. However, the regulatory mechanism of NEAT1 in AOM remains unclear and must be further investigated. Therefore, the present study focused on the biological function of NEAT1 in AOM.

MicroRNAs (miRNAs/miRs) are non-coding, endogenous single-stranded small RNAs. Studies have shown that miRNAs may serve as nodes of signaling networks, participating in the regulation of basic cell activities, such as cell inflammation (19). miR-301b-3p was found to be significantly reduced in LPS-induced human middle ear epithelial cells (HMEECs) (20), but the biological function of miR-301b-3p in AOM has not been reported to date. Furthermore, miRNAs can negatively regulate protein expression by targeting the 3'-untranslated region (UTR) of their target mRNAs (21). Based on bioinformatics prediction, the toll-like receptor 4 gene (TLR4) was shown to be a target of miR-301b-3p in the present study. TLR4 has been proven to play an essential role in triggering the inflammatory response, and TLR4 signal activation promotes an increase in the production and upregulation of pro-inflammatory factors $(22,23)$. Furthermore, the TLR4 protein was found to be upregulated by non-typeable $H$. influenzae infection and LPS induction in animal models and patients with AOM (24-27). Hence, it was hypothesized that miR-301b-3p and TLR4 may be involved in NEAT1-mediated inflammation in AOM. The aim of the present study was to investigate the effect of NEAT1 on inflammatory response in LPS-treated HMEECs and explore the potential interactions among NEAT1, miR-301b-3p and TLR4.

\section{Materials and methods}

Cell culture and LPS treatment. HMEECs were purchased from American Type Culture Collection; human renal epithelial cells (HK2) and human liver epithelial cells (THLE-3) were purchased from Beijing Beina Chuanglian Institute of Biotechnology. HMEECs and THLE-3 cells were routinely cultured in RPMI-1640 medium supplemented with 10\% FBS (both from Gibco; Thermo Fisher Scientific, Inc.) and $1 \%$ penicillin/streptomycin. HK2 cells were cultured in DMEM (Gibco; Thermo Fisher Scientific, Inc.) supplemented with $10 \% \mathrm{FBS}, 100 \mathrm{U} / \mathrm{ml}$ penicillin and $100 \mu \mathrm{g} / \mathrm{ml}$ streptomycin. The cells were kept in a humidified atmosphere with $5 \% \mathrm{CO}_{2}$ and $95 \%$ humidity at $37^{\circ} \mathrm{C}$ in an incubator. HMEECs were stimulated with LPS (Sigma-Aldrich; Merck KGaA) at different concentrations $(0,0.1,1,3,5$ and $10 \mu \mathrm{g} / \mathrm{ml})$ for $24 \mathrm{~h}$, and then cultured for 12,24 and $48 \mathrm{~h}$ to determine cell viability. The cells were incubated with $5 \mu \mathrm{g} / \mathrm{ml}$ LPS for 6,12 ,
24 and $48 \mathrm{~h}$ to determine the optimal action time. Finally, $5 \mu \mathrm{g} / \mathrm{ml}$ LPS was used for $24 \mathrm{~h}$ to establish an inflammatory cell model.

Cell transfection. Transfections were performed using $1.0 \mu \mathrm{g}$ small interfering (si)RNA of NEAT1 (si-NEAT1; 5'-GUGAGA AGUUGCUUAGAAACUUUCC-3'), $1.0 \mu \mathrm{g}$ si-TLR4 (5'-TTGC TAAATGCTGCCGTTTTATC-3'), $1.0 \mu \mathrm{g}$ si-RNA-negative control (si-NC; 5'-UACUGUCUAGUCGCCGUAC-3'), $100 \mathrm{nM}$ miR-301b-3p mimic (5'-CAGUGCAAUGAUAUUGUCAA AGC-3'), 100 nM NC mimic (5'-UUGUACUACACAAAAGU ACUG-3'), 100 nM miR-301b-3pinhibitor (5'-GCUUUGACAA UAUCAUUGCACUG-3') and $100 \mathrm{nM} \mathrm{NC}$ inhibitor (5'-CAG UACUUUUGUGUAGUACAA-3'), which were obtained from Shanghai GenePharma Co., Ltd.. To overexpress NEAT1 or $T L R 4$, the full-length sequence was inserted into the pcDNA3.1 vector ( $2 \mu \mathrm{g}$; Shanghai GenePharma Co., Ltd.), and an empty vector $(2 \mu \mathrm{g})$ was used as a control. HMEECs cells $\left(1 \times 10^{5}\right)$ were transfected with si-RNA, miR-mimic, miR-inhbitor, overexpression vectors and corresponding NCs using Lipofectamine $^{\circledR} 2000$ (Invitrogen; Thermo Fisher Scientific, Inc.) at $37^{\circ} \mathrm{C}$. After transfection for $48 \mathrm{~h}$, the efficiency of transfection was detected by reverse transcription-quantitative PCR (RT-qPCR) and western blot analysis.

$R N A$ extraction and $R T-q P C R$. Total RNA was extracted from cells using TRIzol ${ }^{\circledR}$ reagent (Invitrogen; Thermo Fisher Scientific, Inc.), according to the manufacturer's protocol. The first cDNA of total RNA was synthesized according to the instructions of PrimeScript ${ }^{\mathrm{TM}}$ RT reagent kit (Takara Biotechnology Co., Ltd.). qPCR was subsequently performed using the SYBR Green qPCR kit (Thermo Fisher Scientific, Inc.), according to the manufacturer's protocols. The following primer sequences were used for RT-qPCR: Taurine upregulated gene 1, forward 5'-AAATCTGGCTCTGCTGACCC-3' and reverse 5'-CAGAAAGGCCAGCCATACCA-3'; zinc finger antisense 1 , forward 5'-AACCATTAGCTAGCTGG GGC-3' and reverse 5'-CAAGTTAACCCCGGAGGGAC-3'; plasmacytoma variant translocation 1, forward 5'-CCTGTGA CCTGTGGAGACAC-3' and reverse 5'-GTCCGTCCAGAGT GCTGAAA-3'; zinc finger E-box binding homeobox 1 antisense 1, forward 5'-CCGTGGGCAC-TGCTGAAT-3' and reverse 5'-CTGCTGGCAAGCGGAACT-3'; X-inactive-specific transcript, forward 5'-CTTGGATGGGTTGCCAGCTA-3' and reverse 5'-TCATGCCCCATCTCCACCTA-3'; NEAT1, forward 5'-CAGTTAGTTTATCAG-TTCTCCCATCCA-3' and reverse 5'-GTTGTTGTCGTCACCTTTCAACTCT-3'; miR-301b-3p, forward 5'-TGCTGCTAACGAATGCTCTGA-3' and reverse 5'-CTCTGCTTTCAGATGCTTTGAC-3'; GAPDH, forward 5'-AGAAGGCTGGGGCTCATTTG-3' and reverse 5'-AGGG GCCATCCACAGTCTTC-3'; and U6, forward 5'-CTCGCTTC GGCAGCACATA-3' and reverse 5'-AACGATTCACGAATT TGCGT-3'. RT-qPCR experiments were performed with the 7900HT Fast Real-time PCR system (Applied Biosystems; Thermo Fisher Scientific, Inc.). The following thermocy-cling conditions were used for qPCR: Initial denaturation at $95^{\circ} \mathrm{C}$ for $7 \mathrm{~min}$, followed by 40 cycles of denaturation at $95^{\circ} \mathrm{C}$ for $10 \mathrm{sec}$, annealing at $60^{\circ} \mathrm{C}$ for $20 \mathrm{sec}$ and elongation at $72^{\circ} \mathrm{C}$ for $20 \mathrm{sec}$, with final extension at $72^{\circ} \mathrm{C}$ for $10 \mathrm{~min}$. Relative expression levels were calculated using the $2^{-\Delta \Delta \mathrm{Cq}}$ method (28) and 
normalized to the internal reference genes GAPDH (mRNA) and U6 (miRNA).

Cell viability assay. Cell viability was assessed using the Cell Counting Kit- 8 proliferation de-tection kit (Beyotime Institute of Biotechnology). Briefly, cells were seeded in 96-well plates at a density of $10^{4}$ cells/well; $10 \mu \mathrm{l}$ CCK-8 solution was added to each well and cultured for $4 \mathrm{~h}$. The OD value at $450 \mathrm{~nm}$ was measured with a microplate reader (BioTek Instruments, Inc.).

Detection of cell apoptosis. Apoptosis was detected using an Annexin V-fluorescein isothiocyanate (FITC)/propidium iodide (PI) cell apoptosis detection kit (Beijing Solarbio Science \& Technology Co., Ltd.). In brief, HMEECs were collected using cold PBS and then cultured with $5 \mu$ Annexin V-FITC reagent and $5 \mu \mathrm{l} \mathrm{PI}$ in the dark for $15 \mathrm{~min}$. The cell apoptosis rate was analyzed by flow cytometry (BD Biosciences) and FlowJo software (version 10; FlowJo LLC).

ELISA. Cell-free culture supernatant was collected after treatment. The secretory levels of IL-6 (cat. no. 900-T16), TNF- $\alpha$ (cat. no. 900-M25) and IL-1 $\beta$ (cat. no. 900-M95) were analyzed using their corresponding ELISA kits (Neobioscience Technology Co., Ltd.) according to the manufacturers' instructions. The concentration of inflammatory cytokines was measured at $450 \mathrm{~nm}$ using a microplate spectrophotometer (BioTek Instruments, Inc.).

Western blot analysis. Cells were lysed with RIPA buffer [50 mM Tris- $\mathrm{HCl}(\mathrm{pH} 8.0), 50 \mathrm{mM} \mathrm{NaCl}$ and $0.5 \%$ NP-40]. The total protein $(20 \mu \mathrm{g} / \mathrm{well})$ in the supernatant was separated by $10 \%$ SDS-PAGE and then transferred to PVDF membranes. After blocking with 5\% skimmed milk at room temperature for $2 \mathrm{~h}$, the membrane was incubated with primary antibodies against TLR4 (dilution 1:1,000; cat. no. ab13556; Abcam) or GAPDH (dilution 1:1,000; cat. no. ab8245; Abcam) at $4^{\circ} \mathrm{C}$ overnight, and then incubated with horseradish peroxidase-conjugated secondary antibody (dilution 1:4,000; cat. no. sc-2357; Santa Cruz Biotechnology, Inc.) for $2 \mathrm{~h}$ at room temperature. The immunoreactive bands were visualized using an enhanced chemilumi-nescence reagent (Beyotime Institute of Biotechnology). The blots were semiquantified by ImageJ software, version 1.47 (National Institutes of Health).

Bioinformatics and luciferase reporter analysis. The starBase (version 2.0: star-base.sysu.edu.cn) online software was used to predict targeting binding sites of lncRNA to miRNA and miRNA to mRNA. Therefore, the targeting associations between miR-301b-3p and NEAT1 or TLR4 were predicted. Reporter vectors were constructed using pmirGLO dual-luciferase expression vector (Promega Corporation), including wild-type (WT) and mutant (MUT) binding sequences of NEAT1 and TLR4, named as WT-NEAT1, MUT-NEAT1, WT-TLR4 and MUT-TLR4, and were co-transfected with miR-301b-3p mimics or negative control (sequences as aforementioned) into 293 cells (The Cell Bank of Type Culture Collection of the Chinese Academy of Sciences, Shanghai, China) using Lipofectamine ${ }^{\circledR} 2000$ (Invitrogen; Thermo Fisher Scientific, Inc.) at $37^{\circ} \mathrm{C}$. After $48 \mathrm{~h}$ of transfection, luciferase activities were measured with a dual-luciferase reporter assay system (Promega Corporation). Firefly luciferase activity was normalized to Renilla luciferase activity and all experiments were performed in triplicate.

Statistical analysis. All experiments were repeated three times. GraphPad Prism 8 (GraphPad Software, Inc.) was used for statistical analysis, and data are presented as mean \pm standard deviation. Differences between the two groups were analyzed using unpaired Student's t-tests and differences between multiple groups data were analyzed by one-way ANOVA followed by Tukey's post hoc tests. $\mathrm{P}<0.05$ was considered to indicate statistically significant differences.

\section{Results}

LPS induces apoptosis and inflammatory response in HMEECs and inhibits cell viability. To investigate whether LPS causes HMEEC injury, different concentrations $(0,0.1$, $1,3,5$ and $10 \mu \mathrm{g} / \mathrm{ml}$ ) of LPS were used to treat HMEECs for 12,24 and $48 \mathrm{~h}$. The CCK- 8 assay demonstrated that the viability of HMEECs was inhibited after LPS induction in a time- and concentration-dependent manner (Fig. 1A). Following treatment with 3 and $5 \mu \mathrm{g} / \mathrm{ml}$ for $48 \mathrm{~h}$, the cell viability decreased by $\sim 50 \%(\mathrm{P}<0.05)$, and it was significantly decreased in the $5 \mu \mathrm{g} / \mathrm{ml}$ group compared with that in the $0 \mu \mathrm{g} / \mathrm{ml}$ (control) group ( $\mathrm{P}<0.01$; Fig. 1B). Therefore, HMEECs were treated with $5 \mu \mathrm{g} / \mathrm{ml}$ for $24 \mathrm{~h}$ in the subsequent experiments. LPS-induced HMEEC apoptosis was significantly increased $(\mathrm{P}<0.01$; Fig. $1 \mathrm{C})$, and the concentration of inflammatory factors (IL-6, IL-1 $\beta$ and TNF- $\alpha$ ) was also significantly increased $(\mathrm{P}<0.001$; Fig. 1D), indicating that LPS induced an inflammatory response in HMEECs.

NEAT1 knockdown alleviates cell apoptosis and inflammatory response in LPS-induced HMEECs. RT-qPCR was conducted to explore the possible involvement of lncRNAs in the regulation of AOM-related mechanisms. As shown in Fig. 2A, all six inflammation-related lncRNAs were found to be upregulated in LPS-stimulated HMEECs, among which NEAT1 exhibited the most prominent increase $(\mathrm{P}<0.001)$. Subsequently, si-NEAT1 was transfected into HMEECs to inhibit the expression of NEAT1. The results demonstrated that NEAT1 was successfully silenced (Fig. 2B; P<0.01). CCK-8 assay, flow cytometry and ELISA were conducted to elucidate the effect of NEAT1 knockdown on the proliferation, apoptosis and inflammation of LPS-stimulated HMEECs (Fig. 2C-E). The results revealed that NEAT1 knockdown relieved the proliferation inhibition of LPS-induced HMEECs $(\mathrm{P}<0.01)$, and markedly alleviated cell apoptosis and the levels of the inflammatory factors IL-6, IL- $1 \beta$ and TNF- $\alpha(\mathrm{P}<0.05$ and $\mathrm{P}<0.001$, respectively). Taken together, these results suggested that the downregulation of NEAT1 inhibited LPS-induced HMEEC inflammatory injury.

miR-301b-3p targets NEAT1 and TLR4 in HMEECs. Epithelial cells are protected from injury due to different stressors through inflammation response regulation. To investigate the abnor-mal expression of miR-301b-3p and TLR4 in LPS-induced epithelial cells, as described by Ma et al (29) in HMEECs, HK-2 and THLE-3 cells were selected, and then exposed to LPS 

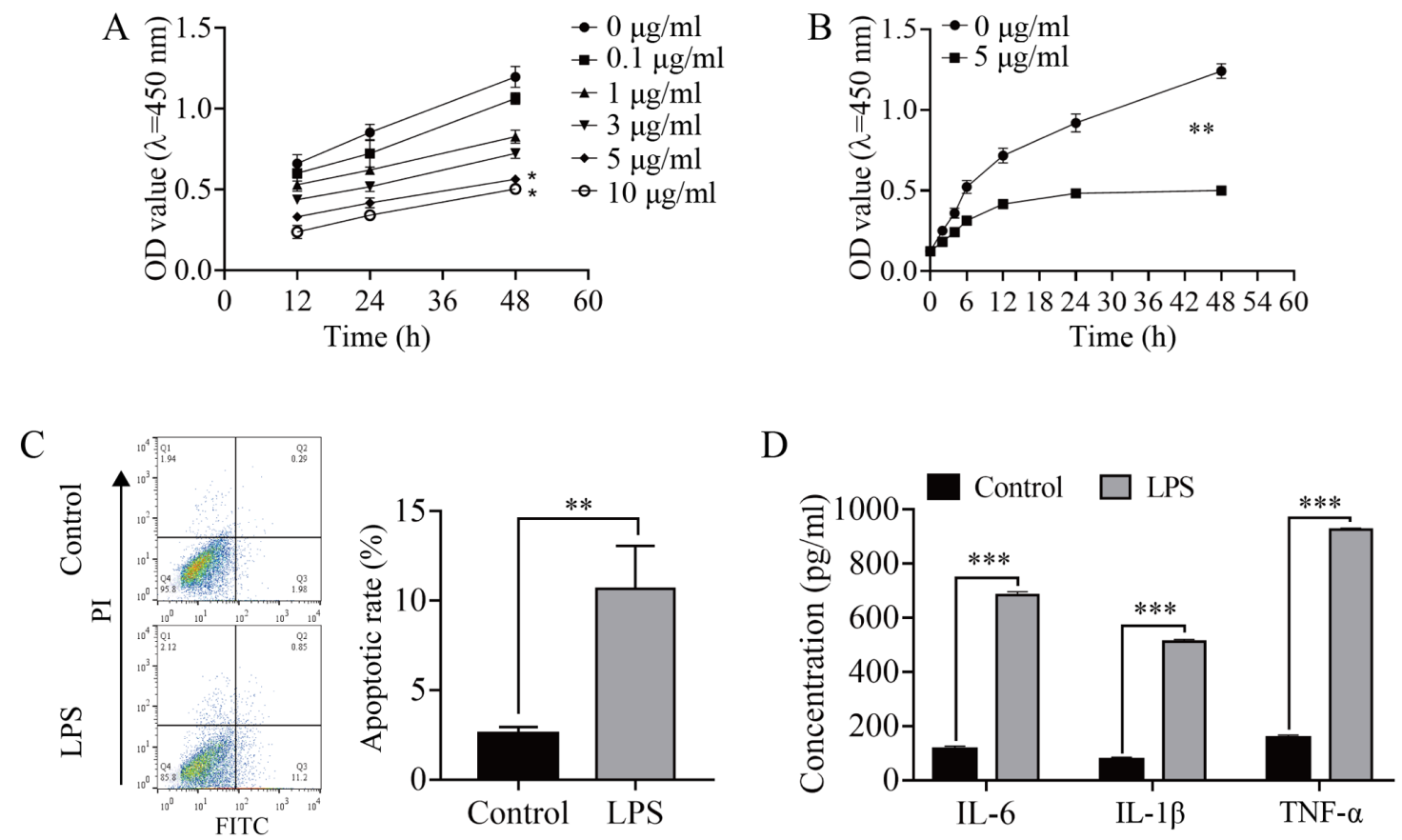

Figure 1. LPS reduces cell viability, and increases apoptosis and inflammatory response in human middle ear epithelial cells. (A and B) Cell viability was estimated by Cell Counting Kit- 8 assay. ${ }^{*} \mathrm{P}<0.05,{ }^{* * *} \mathrm{P}<0.01$ vs. $0 \mu \mathrm{g}$. (C) Cell apoptosis was evaluated by flow cytometry. ${ }^{* *} \mathrm{P}<0.01 \mathrm{vs}$. control. (D) IL-6, IL-1 $\beta$ and TNF- $\alpha$ expression was evaluated by ELISA. ${ }^{* * *} \mathrm{P}<0.001$ vs. control. LPS, lipopolysaccharide; OD, optical density; FITC, fluorescein isothi-ocyanate; PI, propidium iodide.

for 48 h. miR-301b-3p expression in HMEECs was markedly downregulated following LPS treat-ment (Fig. 3A; $\mathrm{P}<0.01$ ), while TLR4 expression was significantly upregulated (Fig. 3B; $\mathrm{P}<0.05)$. Moreover, miR-301b-3p and TLR4 levels in HK-2 and THLE-3 cells, as well as in HMEECs, were detected by RT-qPCR and western blot assays. miR-301b-3p and TLR4 levels were down- and upregulated, respectively, following LPS induction (Fig. 3A and $\mathrm{B} ; \mathrm{P}<0.05$ ). These results suggested that LPS down- and upregulated miR-301b-3p and TLR4 levels, re-spectively, in HMEECs. A search through the bioinformatics database starBase revealed a putative interaction between NEAT1 and miR-301b-3p (the target binding sequence is shown in Fig. 3C). Dual-luciferase reporter assay demonstrated that NEAT1-WT and miR-301b-3p mimics cotransfection significantly decreased luciferase activity in HMEECs, while NEAT1-MUT and miR-301b-3p mimics cotransfection failed to affect luciferase activity in HMEECs (Fig. 3D; P<0.01). Furthermore, NEAT1 silencing significantly increased miR-301b-3p expression (Fig. 3E; P<0.01). These results indicated that miR-301b-3p was targeted by NEAT1. Subsequently, based on the biological information database starBase, the target gene of miR-301b-3p was predicted, and it was found that TLR4, which is involved in inflammation, and miR-301b-3p had a targeted binding sequence (Fig. 3F). Dual-luciferase reporter assay demonstrated that the luciferase activity of TLR4-WT was decreased by miR-301b-3p mimics in HMEECs, while TLR4-MUT and miR-301b-3p mimics cotransfection failed to affect luciferase activity (Fig. 3G; $\mathrm{P}<0.01)$. Subsequently, pc-NEAT1 vector and miR-301b-3p mimic were transfected into HMEECs to upregulate the expression of NEAT1 and miR-301b-3p, and the results revealed that NEAT1and miR-301b-3p expression was strongly enhanced (Fig. 3H and 3I; $\mathrm{P}<0.01$ ). As shown in Fig. 3J, overexpression of miR-301b-3p inhibited TLR4 expression, which was reversed by overexpressing TLR4 (Fig. 3J; P<0.05). Taken together, these results proved that miR-301b-3p directly targets both NEAT1 and TLR4.

NEAT1 regulates LPS-induced proliferation, apoptosis and inflammation of HMEECs via the miR-301-3p/TLR4 axis. To explore how NEAT1 regulates LPS-induced HMEEC proliferation, inflammation and apoptosis, miR-301b-3p inhibitors, si-NEAT1, si-TLR4 and pc-TLR4 were transfected into HMEECs to detect their regulatory effects on inflammation. Silencing miR-301b-3p significantly decreased miR-301b-3p expression compared with the $\mathrm{NC}$ group (Fig. 4A; $\mathrm{P}<0.01$ ), and then transfection of si-TLR4 and pc-TLR4 resulted in effective decrease and increase in the expression of TLR4, respectively (Fig. 4B and $\mathrm{C} ; \mathrm{P}<0.01$ ). The $\mathrm{CCK}-8$ assay revealed that LPS inhibited cell proliferation, which was recovered with si-NEAT1 and si-TLR4, but proliferation was finally suppressed by miR-301b-3p inhibitors or overexpression TLR4 (Fig. 4D). Similarly, the inflammation and apoptosis of HMEECs was analyzed. Flow cytometry and ELISA revealed an opposite trend than the CCK-8 assay (Fig. 4E and F). Next, western blotting was performed to verify whether NEAT1 regulated the miR-301-3p/TLR4 axis and protein expression of TLR4. As shown in Fig. 4G, miR-301b-3p inhibitors or overexpression of TLR4 abolished the inhibitory effect of NEAT1 silencing on the protein expression of TLR4. In addition, miR-301b-3p inhibitors reversed the suppression of the protein expression of TLR4 by TLR4 silencing. Taken together, these results indicated that NEAT1 attenuated LPS-induced inflammation in HMEECs via modulating the miR-301b-3p/TLR4 axis. 

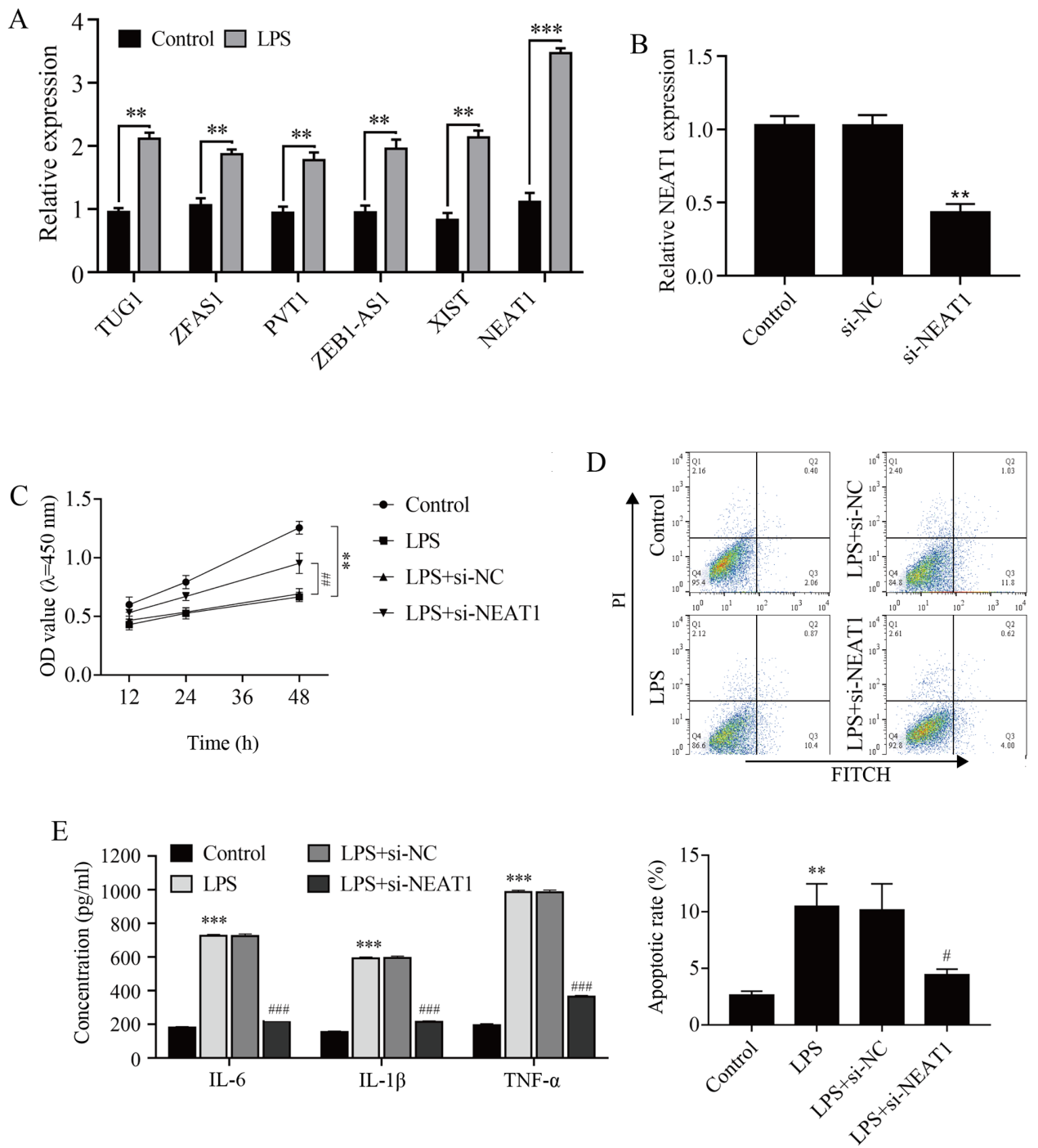

Figure 2. NEAT1 knockdown alleviates cell apoptosis and inflammatory response in LPS-induced human middle ear epithelial cells. (A) The expression of different long non-coding RNAs and (B) transfection efficiency of si-NEAT1 were analyzed by reverse transcription-quantitative PCR. (C) Cell viability was analyzed by Cell Counting Kit-8 assay. (D) Cell apoptosis was evaluated by flow cytometry. (E) IL-6, IL-1 $\beta$ and TNF- $\alpha$ expression was measured by ELISA. ${ }^{* *} \mathrm{P}<0.01$ and ${ }^{* * *} \mathrm{P}<0.001$ vs. control; ${ }^{~} \mathrm{P}<0.05,{ }^{\# \#} \mathrm{P}<0.01$ and ${ }^{\# \# "} \mathrm{P}<0.001$ vs. LPS+si-NC. LPS, LPS-stimulated; NEAT1, nuclear enriched abundant transcript 1; TUG1, taurine upregulated gene 1; ZFAS1, zinc finger antisense 1; PVT1, plasmacytoma variant translocation 1; ZEB1-AS1, zinc finger E-box binding homeobox 1 antisense 1; XIST, X-inactive-specific transcript; LPS, lipopolysaccharide; NC, negative control; si, small interfering; OD, optical density; FITC, fluorescein isothiocyanate; PI, propidium iodide.

\section{Discussion}

$\mathrm{AOM}$ is one of the most common infectious diseases in children. Its pathological characteristic is inflammation, with induced secretion of cytokines TNF- $\alpha$, IL-6 and IL-8 (30). AOM may develop into recurrent or persistent otitis media, which causes progressive hearing loss and severely affects the quality of life of the patients (1), even causing language disability and intellectual impairment (31). The specific molecular mechanism of AOM is poorly understood and finding effective therapeutic strategies is urgently required. Therefore, the present study aimed to investigate the NEAT1/miR-301b-3p/TLR4 regulatory network, in the hope of revealing a new theoretical basis for the application of NEAT1 in the treatment of AOM. In the present study,LPS was used to establish the cell model of AOM and investigate the potential mechanism $(26,32)$. According to previous reports, NEAT1 is induced in multiple immune system diseases, such as pneumonia (15), hepatitis (17) and osteoarthritis (33). Thus, it was hypothesized that NEAT1 may be induced in LPS-stimulated HMEECs. In the present study, NEAT1 was found to be significantly increased in LPS-induced HMEECs, and knockdown of NEAT1 significantly improved cell proliferation and inhibited cell apoptosis and inflammation. These data suggested that NEAT1 knockdown attenuated LPS-induced inflammation and apoptosis.

NEAT1 has been previously found to be induced in various tumors (13). For example, NEAT1 was shown to increase cell survival and facilitate gallbladder cancer progression by sponging miR-335 (34). Furthermore, NEAT1 is not only im-plicated in cancer, but may also affect the inflammatory response. Li et al (35) reported that NEAT1 may promote LPS-induced inflammatory injury in macrophages by regulating miR-17-5p/TLR4. In addition, downregulation of NEAT1 promoted cell proliferation and inhibited the apoptosis of rat cardiac muscle cells (36). However, to the best 


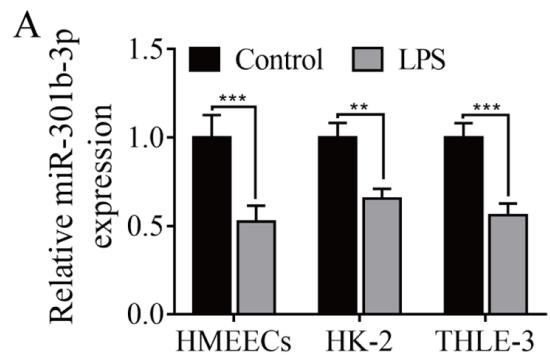

C

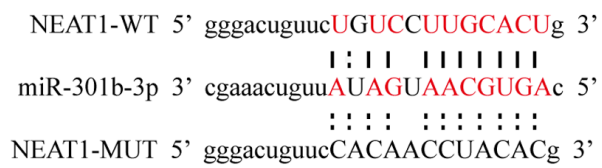

F

TLR4-WT 5, aacaauACUGUAUU--AUGCACUu 3,

$$
\text { I।:I।I: I। III }
$$

miR-301b-3p 3' cgaaacUGUUAUAGUAACGUGAc 5'

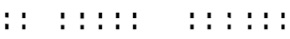

TLR4-MUT 5', aacaauGACACGCC--GCAAGACg 3'

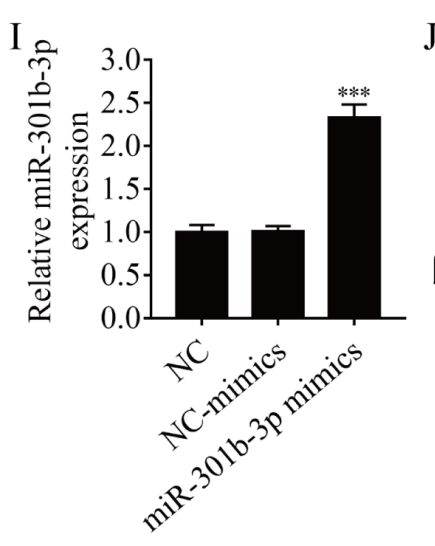

B
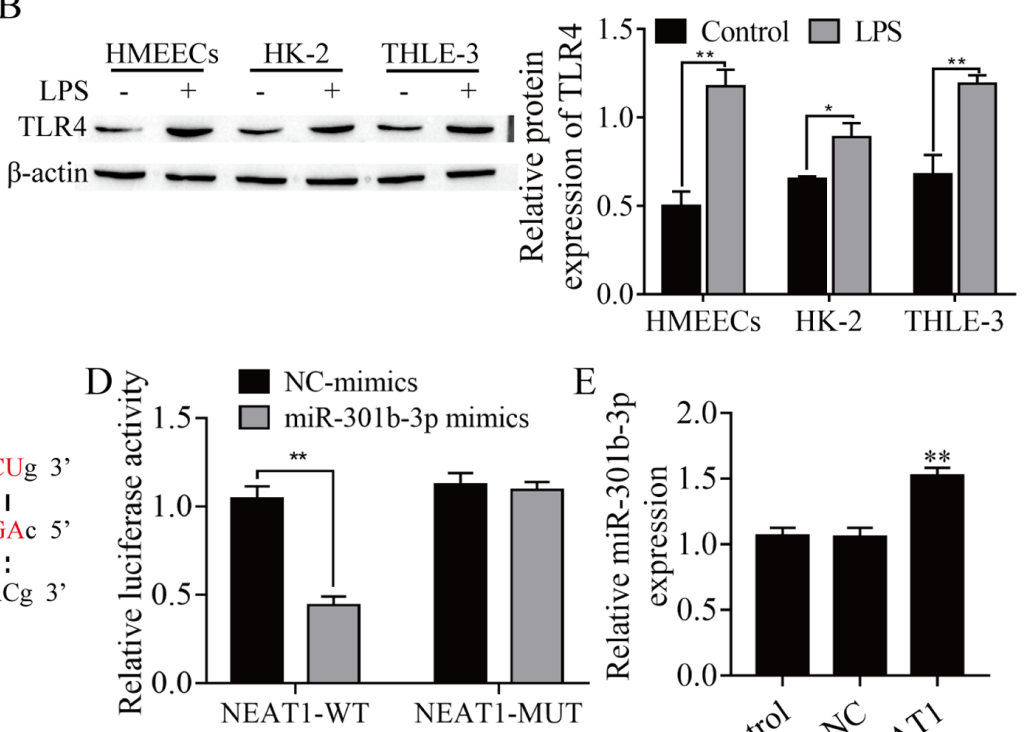

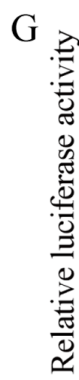

$\mathrm{H}$

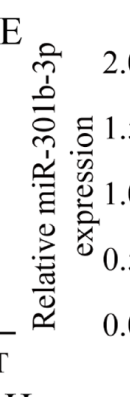

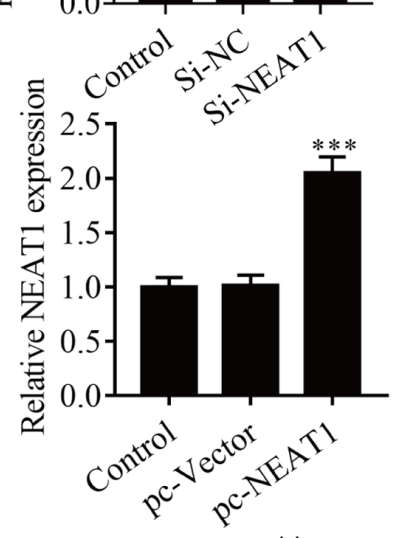

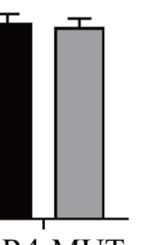

TLR4-WT TLR4-MUT
$\mathrm{J}$

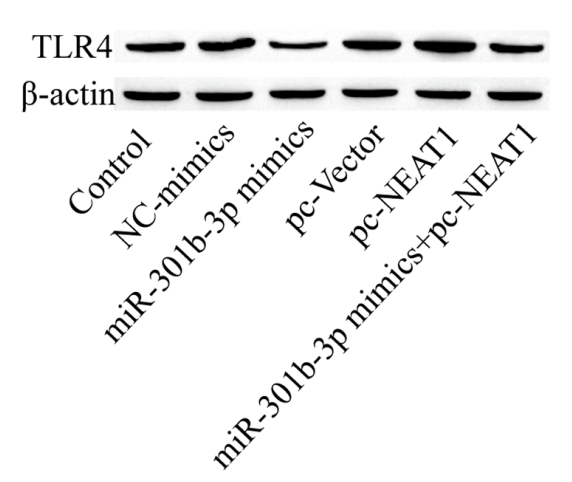

章 

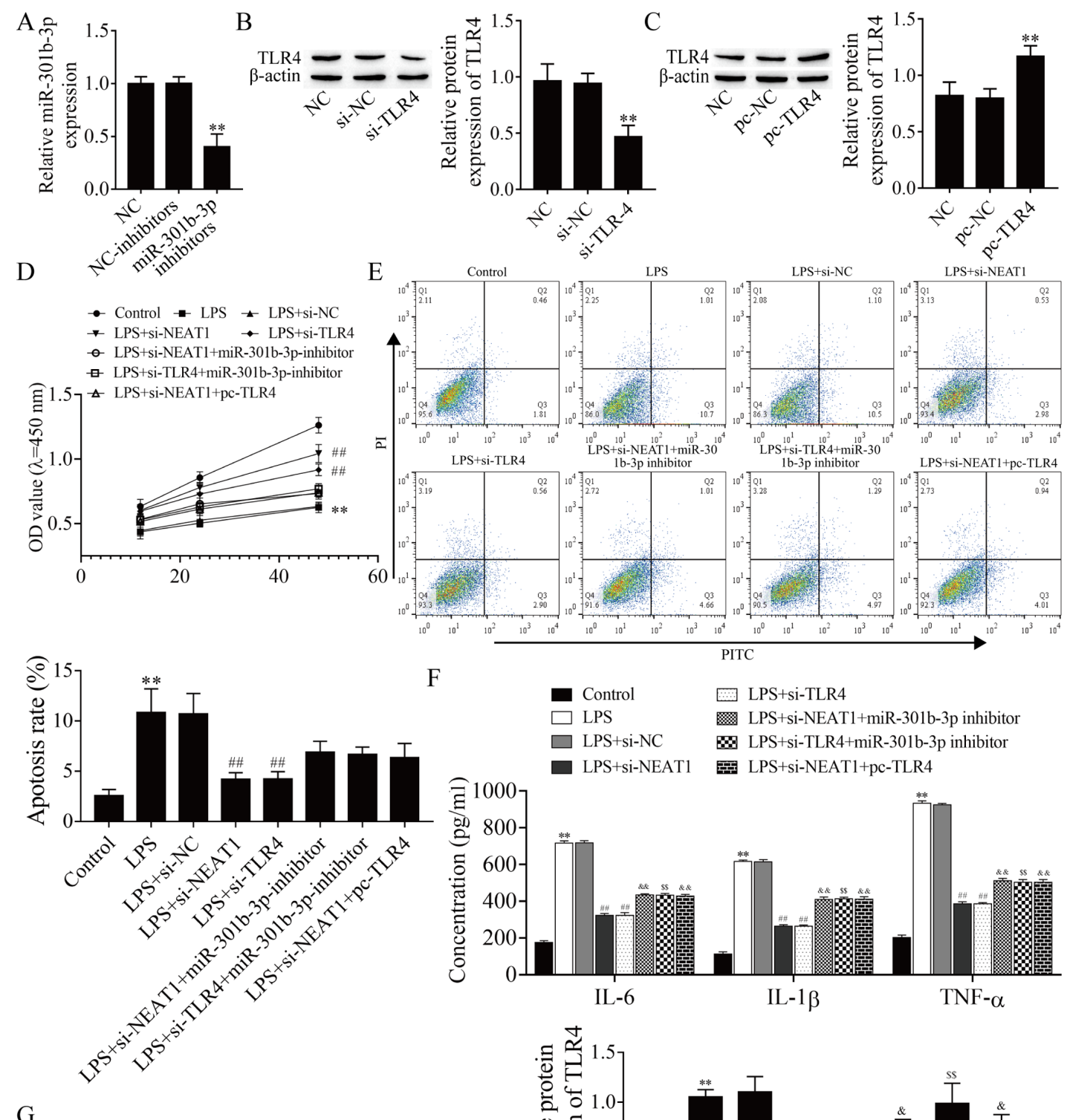

G
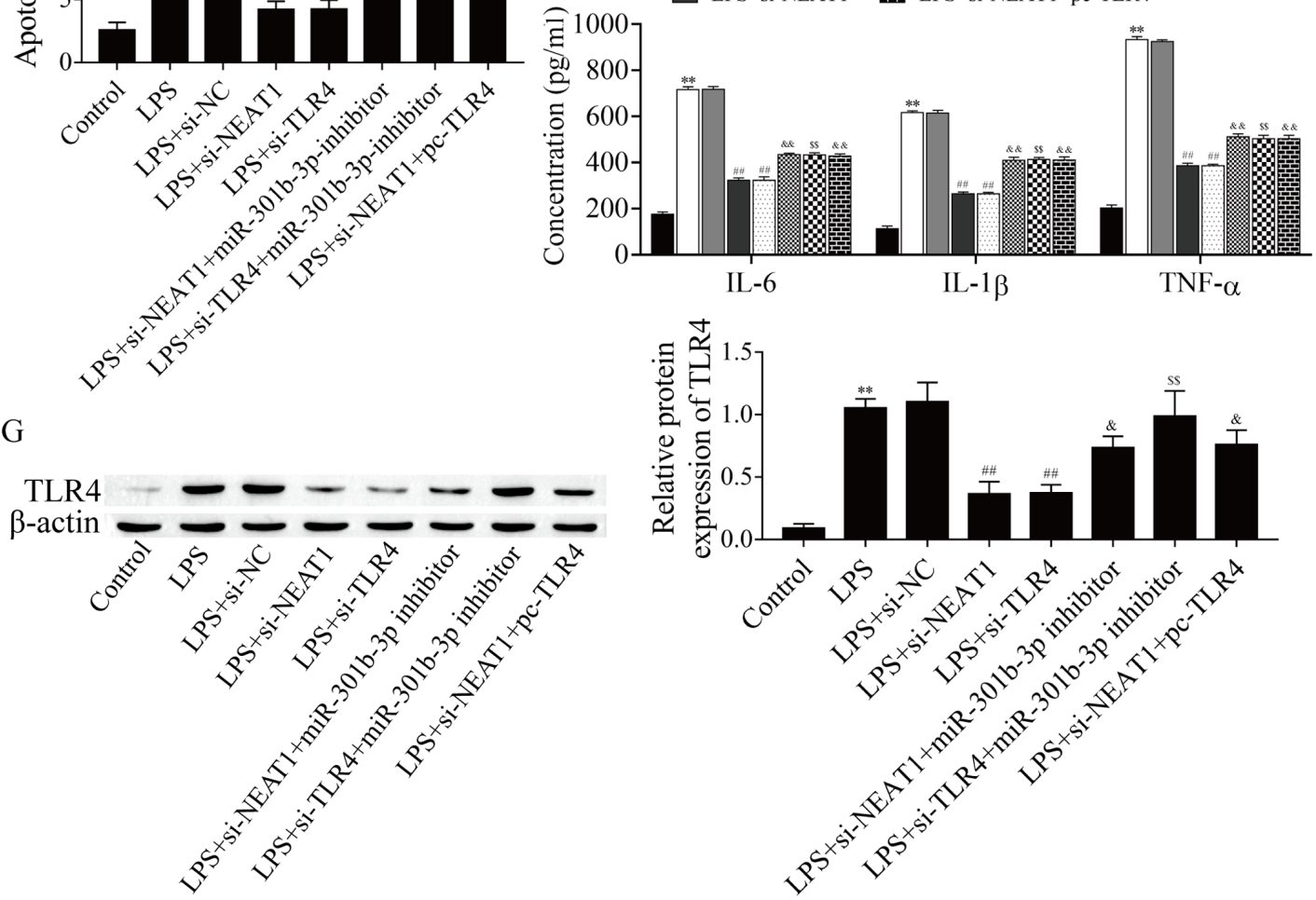

Figure 4. NEAT1 regulates LPS-induced cell viability, apoptosis and inflammation of human middle ear epithelial cells via the miR-370-3p/TLR4 axis. (A) miR-301b-3p expression was determined by reverse transcription-quantitative PCR. TLR4 protein expression was detected by western blot assay after transfection with (B) si-TLR4 and (C) pc-TLR4. (D) Cell viability was analyzed by Cell Counting Kit-8 assay. (E) Cell apoptosis was evaluated by flow cytometry. (F) IL-6, IL-1 $\beta$ and TNF- $\alpha$ expression levels were detected by ELISA. (G) TLR4 protein expression in different groups was detected by western blot assay. ${ }^{* * *} \mathrm{P}<0.01$ vs. control, si-NC or pc-NC; ${ }^{\# \#} \mathrm{P}<0.01$ vs. control and $\mathrm{LPS}+\mathrm{si}-\mathrm{NC}$; ${ }^{\circledR} \mathrm{P}<0.05$ vs. LPS+si-NEAT1; ${ }^{\& \&} \mathrm{P}<0.01$ vs. LPS+si-NEAT1; ${ }^{\$ \$} \mathrm{P}<0.01$ vs LPS+si-TLR4. LPS, lipopolysaccharide; miR, microRNA; NC, negative control; si, small interfering; NEAT1, nuclear enriched abundant transcript 1; TLR4, toll-like receptor 4; OD, optical density; FITC, fluorescein isothiocyanate; PI, propidium iodide.

downregulated and determined to be a target of and negatively regulated by NEAT1, thereby inhibiting LPS-induced apoptosis and inflammation of HMEECs. According to previous reports, miR-301b-3p was shown to contribute to tumor growth in human hepatocellular carcinoma and gastric cancer $(38,39)$. In addition, the expression of miR-301b-3p was significantly 
upregulated by Pseudomonas aeruginosa and Candida albicans $(22,40,41)$, but miR-301b-3p was downregulated in LPS-induced HMEECs (20). miR-301b-3p and TLR4 expression was also investigated in the HK2 and THLE-3 cells. The results demonstrated that the expression of miR-301b-3p was reduced in LPS-induced HK2 and THLE-3 cells, whereas TLR4 expression was increased. The present study found that miR-301b-3p was a target of NEAT1, and miR-301b-3p inhibitors reversed the inhibitory effect of NEAT1 silencing on the inflammatory response of HMEECs. In this context, our data suggested that NEAT1 may regulate LPS-induced inflammation of HMEECs by sponging miR-301b-3p.

Previous studies have reported that TLR4 is highly expressed in necrotizing enterocolitis (42), oral cancer and brain injury (43). Of note, TLR4 is also highly expressed in $\mathrm{AOM}(31,44)$. Consistent with the aforementioned findings, it was observed herein that the enrichment of TLR4 increased in LPS-stimulated HMEECs compared with normal HMEECs. In addition, miRNAs can regulate gene expression through binding to the 3'-UTR of their target mRNAs (45). For example, miR-301b-3p targets the 3'-UTR of PTEN and promotes cell migration and invasion in bladder cancer (46). Previous studies reported that miR-301b-3p was transcriptionally upregulated by the TLR4/NF- $\kappa \mathrm{B}$ axis in human bladder cancer and Candida albicans-induced lung injury $(40,47)$. Notably, in patients with AOM, the expression of TLR4 was upregulated, and H. influenzae inoculation and LPS-induced inflammation also upregulated the TLR4 level in animal models with AOM (24-27), but LPS induction significantly reduced $\mathrm{miR}-301 \mathrm{~b}-3 \mathrm{p}$ expression in HMEECs (20). It was also observed that LPS induction significantly increased TLR4 and decreased miR-301b-3p expression in HMEECs. Notably, TLR4 was found to be the target gene of miR-301b-3p, and miR-301b-3p inhibited TLR4 expression in LPS-induced HMEECs. Li et al (35) reported that NEAT1 may promote LPS-induced inflammatory injury in macrophages by regulating miR-17-5p/TLR4. Consistently, the present study found that miR-301b-3p inhibitors and TLR4 overexpression upregulated the inhibitory effect of NEAT1 silencing on TLR4, indicating that NEAT1 affects TLR4 expression by modulating miR-301b-3p, thereby promoting LPS-induced apoptosis and inflammation of HMEECs. However, the present study only investigated the potential role and mechanism of the NEAT1/miR-301b-3p/TLR4 axis in LPS-induced HMEECs, and several limitations should also be considered. Preclinical data are expected to improve our understanding of the potential mechanism by using mouse models in vivo or primary cells in vitro in future studies.

In summary, it was herein demonstrated that interference with lncRNA NEAT1 expression may protect HMEECs from LPS-induced inflammation and apoptosis via the miR-301b-3p/TLR4 axis, which contributes to improving the pathological status of AOM. The findings of the present study also indicated that the NEAT1/miR-301b-3p/TLR4 axis may constitute a potential therapeutic target for AOM.

\section{Acknowledgements}

Not applicable.

\section{Funding}

The present study was supported by the National Natural Science Foundation of China (grant no. 81960187).

\section{Availability of data and materials}

The datasets used and/or analyzed during the present study are available from the corresponding author on reasonable request.

\section{Authors' contributions}

ZL and RL conceived and designed the research. TL, SL, FZ, JY, SD and BR performed the experiments and analyzed the data. ZL, TL and SL wrote the manuscript. ZL and RL interpreted the data. RL, BR and RL confirm the authenticity of all the raw data. All the authors have read and approved the final manuscript.

\section{Ethics approval and consent to participate}

Not applicable.

\section{Patient consent for publication}

Not applicable.

\section{Competing interests}

The authors declare that they have no competing interests.

\section{References}

1. Morris LM, DeGagne JM, Kempton JB, Hausman F and Trune DR: Mouse middle ear ion homeostasis channels and intercellular junctions. PLoS One 7: e39004, 2012.

2. Block SL: Causative pathogens, antibiotic resistance and therapeutic considerations in acute otitis media. Pediatr Infect Dis J 16: 449-456, 1997.

3. Post JC: Direct evidence of bacterial biofilms in otitis media. 2001. Laryngoscope 125: 2003-2014, 2015.

4. Topcuoglu N, Keskin F, Ciftci S, Paltura C, Kulekci M, Ustek D and Kulekci G: Relationship be-tween oral anaerobic bacteria and otitis media with effusion. Int J Med Sci 9: 256-261, 2012.

5. 5. Beermann J, Piccoli MT, Viereck J and Thum T: Non-coding RNAs in development and disease: Background, mechanisms, and therapeutic approaches. Physiol Rev 96: 1297-1325, 2016.

6. Curtale G and Citarella F: Dynamic nature of noncoding RNA regulation of adaptive immune response. Int J Mol Sci 14: 17347-17377, 2013.

7. Wang J, Lucas BA and Maquat LE: New gene expression pipelines gush lncRNAs. Genome Biol 14: 117, 2013.

8. Zhang Y, Zhu Y, Gao G and Zhou Z: Knockdown XIST alleviates LPS-induced WI-38 cell apoptosis and inflammation injury via targeting miR-370-3p/TLR4 in acute pneumonia. Cell Biochem Funct 37: 348-358, 2019.

9. Liang WJ, Zeng XY, Jiang SL, Tan HY, Yan MY and Yang HZ: Long non-coding RNA MALAT1 sponges miR-149 to promote inflammatory responses of LPS-induced acute lung in-jury by targeting MyD88. Cell Biol Int 44: 317-326, 2020.

10. Gomez JA, Wapinski OL, Yang YW, Bureau JF, Gopinath S, Monack DM, Chang HY, Brahic M and Kirkegaard K: The NeST long ncRNA controls microbial susceptibility and epigenetic activation of the interferon- $\gamma$ locus. Cell 152: 743-754, 2013.

11. Pawar K, Hanisch C, Palma Vera SE, Einspanier R and Sharbati S: Down regulated lncRNA MEG3 eliminates mycobacteria in macrophages via autophagy. Sci Rep 6: 19416, 2016. 
12. Wang Y, Zhong H, Xie X, Chen CY, Huang D, Shen L, Zhang H, Chen ZW and Zeng G: Long noncoding RNA derived from CD244 signaling epigenetically controls $\mathrm{CD}^{+} \mathrm{T}$-cell immune responses in tuberculosis infection. Proc Natl Acad Sci USA 112: E3883-E3892, 2015.

13. Shen X, Zhao W, Zhang Y and Liang B: Long non-coding RNA-NEAT1 promotes cell migration and invasion via regulating miR-124/NF- $\kappa \mathrm{B}$ pathway in cervical cancer. OncoTargets Ther 13: 3265-3276, 2020.

14. Chen XX, Jiang YJ, Zeng T and Li JJ: Overexpression of the long noncoding RNA NEAT1 pro-tects against $\mathrm{As}_{2} \mathrm{O}_{3}$-induced injury of cardiomyocyte by inhibiting the miR-124/NF- $\mathrm{B}$ signaling pathway. Eur Rev Med Pharmacol Sci 24: 1378-1390, 2020

15. Nong W: Long non-coding RNA NEAT1/miR-193a-3p regulates LPS-induced apoptosis and inflammatory injury in WI-38 cells through TLR4/NF- $\kappa B$ signaling. Am J Transl Res 11: 5944-5955, 2019.

16. Ma J, Zhao N, Du L and Wang Y: Downregulation of lncRNA NEAT1 inhibits mouse mesangial cell proliferation, fibrosis, and inflammation but promotes apoptosis in diabetic nephropathy. Int J Clin Exp Pathol 12: 1174-1183, 2019.

17. Jin SS, Lin XF, Zheng JZ, Wang Q and Guan HQ: IncRNA NEAT1 regulates fibrosis and inflammatory response induced by nonalcoholic fatty liver by regulating miR-506/GLI3. Eur Cytokine Netw 30: 98-106, 2019.

18. Wang W and Guo Z: Downregulation of lncRNA NEAT1 ameliorates LPS-induced inflammatory responses by promoting macrophage M2 polarization via miR-125a-5p/TRAF6/TAK1 axis. Inflammation 43: 1548-1560, 2020.

19. Zhou X, Jeker LT, Fife BT, Zhu S, Anderson MS, McManus MT and Bluestone JA: Selective miRNA disruption in T reg cells leads to uncontrolled autoimmunity. J Exp Med 205: 1983-1991, 2008.

20. Song JJ, Kwon SK, Cho CG, Park SW and Chae SW: Microarray analysis of microRNA expression in LPS induced inflammation of human middle ear epithelial cells (HMEECs). Int J Pediatr Otorhinolaryngol 75: 648-651,2011.

21. Hwang HW and Mendell JT: MicroRNAs in cell proliferation, cell death, and tumorigenesis. Br J Cancer 96 (Suppl): R40-R44, 2007.

22. Wree A, McGeough MD, Inzaugarat ME, Eguchi A, Schuster S, Johnson CD, Peña CA, Geis-ler LJ, Papouchado BG, Hoffman HM, et al: NLRP3 inflammasome driven liver injury and fibrosis: Roles of IL-17 and TNF in mice. Hepatology 67: 736-749, 2018

23. Wang YC, Wang PF, Fang H, Chen J, Xiong XY and Yang QW: Toll-like receptor 4 antagonist attenuates intracerebral hemorrhage-induced brain injury. Stroke 44: 2545-2552, 2013

24. Hirano T, Kodama S, Fujita K, Maeda K and Suzuki M: Role of Toll-like receptor 4 in innate immune responses in a mouse model of acute otitis media. FEMS Immunol Med Microbiol 49: 75-83, 2007

25. Leichtle A, Hernandez M, Pak K, Yamasaki K, Cheng CF, Webster NJ, Ryan AF and Wasser-man SI: TLR4-mediated induction of TLR2 signaling is critical in the pathogenesis and resolution of otitis media. Innate Immun 15: 205-215, 2009.

26. Li P, Chen D and Huang Y: Fisetin administration improves LPS-induced acute otitis media in mouse in vivo. Int $\mathrm{J}$ Mol Med 42: 237-247, 2018

27. Guo H, Li M and Xu LJ: Apigetrin treatment attenuates LPS-induced acute otitis media though suppressing inflammation and oxidative stress. Biomed Pharmacother 109: 1978-1987, 2019.

28. Livak KJ and Schmittgen TD: Analysis of relative gene expression data using real-time quantitative PCR and the 2(-Delta Delta C(T)) Method. Methods 25: 402-408, 2001.

29. Ma YK, Chen YB and Li P: Quercetin inhibits NTHi-triggered CXCR4 activation through suppressing IKK $\alpha / N F-\kappa B$ and MAPK signaling pathways in otitis media. Int J Mol Med 42: 248-258, 2018

30. Li GD, Li TL, Liu H and Sun L: Correlation between recovery time of extended high-frequency audiometry and duration of inflammation in patients with acute otitis media. Eur Arch Otorhinolaryngol 277: 2447-2453, 2020.
31. Zhang J, Xu M, Zheng Q, Zhang Y, Ma W and Zhang Z: Blocking macrophage migration inhibitory factor activity alleviates mouse acute otitis media in vivo. Immunol Lett 162A: 101-108, 2014

32. Tuoheti A, Gu X, Cheng X and Zhang H: Silencing Nrf2 attenuates chronic suppurative otitis media by inhibiting pro-inflammatory cytokine secretion through up-regulating TLR4. Innate Immun 27: 70-80, 2021.

33. Wang Z, Hao J and Chen D: Long noncoding RNA nuclear enriched abundant transcript 1(NEAT1) regulates proliferation, apoptosis, and inflammation of chondrocytes via the miR-181a/glycerol-3-phosphate dehydrogenase 1-like (GPD1L) axis. Med Sci Monit 25: 8084-8094, 2019.

34. Yang F, Tang Z, Duan A, Yi B, Shen N, Bo Z, Yin L, Zhu B, Qiu Y and Li J: Long noncoding RNA NEAT1 upregulates survivin and facilitates gallbladder cancer progression by sponging microRNA-335. OncoTargets Ther 13: 2357-2367, 2020.

35. Li Y, Guo W and Cai Y: NEAT1 Promotes LPS-induced inflammatory injury in macrophages by regulating miR-17-5p/TLR4. Open Med (Wars) 15: 38-49, 2020

36. Ren L, Chen S, Liu W, Hou P, Sun W and Yan H: Downregulation of long non-coding RNA nuclear enriched abundant transcript lpromotes cell proliferation and inhibits cell apoptosis by targeting miR-193a in myocardial ischemia/reperfusion injury. BMC Cardiovasc Disord 19: 192, 2019.

37. Karreth FA and Pandolfi PP: ceRNA cross-talk in cancer: When ce-bling rivalries go awry. Cancer Discov 3: 1113-1121, 2013.

38. Fan H, Jin X, Liao C, Qiao L and Zhao W: MicroRNA-301b-3p accelerates the growth of gastric cancer cells by targeting zinc finger and BTB domain containing 4. Pathol Res Pract 215: $152667,2019$.

39. Guo Y, Yao B, Zhu Q, Xiao Z, Hu L, Liu X, Li L, Wang J, Xu Q, Yang L, et al: MicroRNA-301b-3p contributes to tumour growth of human hepatocellular carcinoma by repressing vestigial like family member 4. J Cell Mol Med 23: 5037-5047, 2019.

40. Wu CX, Cheng J, Wang YY, Wang JJ, Guo H and Sun H: Microrna expression profiling of macrophage line RAW264.7 infected by Candida albicans. Shock: 47: 520-530, 2017.

41. Li X, He S, Li R, Zhou X, Zhang S, Yu M, Ye Y, Wang Y, Huang C and $\mathrm{Wu} \mathrm{M}$ : Pseudomonas aeruginosa infection augments inflammation through miR-301b repression of c-Myb-mediated immune activation and infiltration. Nat Microbiol 1: 16132, 2016.

42. Chen Z, Zhang Y, Lin R, Meng X, Zhao W, Shen W and Fan H: Cronobacter sakazakiiinduces necrotizing enterocolitis by regulating NLRP3 inflammasome expression via TLR4. J Med Microbiol 69: 748-758, 2020

43. Zhou K, Cui S, Duan W, Zhang J, Huang J, Wang L, Gong Z and Zhou Y: Cold-inducible RNA-binding protein contributes to intracerebral hemorrhage-induced brain injury via TLR4 signaling. Brain Behav 10: e01618, 2020.

44. Kaur R, Casey J and Pichichero M: Cytokine, chemokine, and Toll-like receptor expression in middle ear fluids of children with acute otitis media. Laryngoscope 125: E39-E44, 2015.

45. Bartel DP: MicroRNAs: Genomics, biogenesis, mechanism, and function. Cell 116: 281-297, 2004.

46. Egawa H, Jingushi K, Hirono T, Ueda Y, Kitae K, Nakata W, Fujita K, Uemura M, Nonomura N and Tsujikawa K: The miR-130 family promotes cell migration and invasion in bladder cancer through FAK and Akt phosphorylation by regulating PTEN. Sci Rep 6: 20574, 2016.

47. Man X, Piao C, Lin X, Kong C, Cui X and Jiang Y: USP13 functions as a tumor suppressor by blocking the NF- $\mathrm{KB}$-mediated PTEN downregulation in human bladder cancer. J Exp Clin Cancer Res 38: 259, 2019.

This work is licensed under a Creative Commons Attribution-NonCommercial-NoDerivatives 4.0 International (CC BY-NC-ND 4.0) License. 